\title{
The Relationship Between High-Output Stomas, Postoperative Ileus, and Readmission After Rectal Cancer Surgery With Diverting Ileostomy
}

\author{
Naa Lee ${ }^{1, \star}$, Soo Young Lee ${ }^{1, \star}$, Chang Hyun $\mathrm{Kim}^{1}$, Han Deok Kwak², Jae Kyun Ju², Hyeong Rok Kim ${ }^{1}$ \\ ${ }^{1}$ Department of Surgery, Chonnam National University Hwasun Hospital, Chonnam National University Medical School, Hwasun, Korea; \\ ${ }^{2}$ Department of Surgery, Chonnam National University Hospital, Chonnam National University Medical School, Gwangju, Korea
}

Purpose: This study aimed to evaluate the relationship between high-output stomas (HOSs), postoperative ileus (POI), and readmission after rectal cancer surgery with diverting ileostomy.

Methods: We included 302 patients with rectal cancer who underwent restorative resection with diverting ileostomy between January 2011 and December 2015. HOSs were defined as stomas with $\geq 2,000 \mathrm{~mL} /$ day output. We analyzed predictive factors for readmission of these patients.

Results: Forty-eight patients (15.9\%) had HOSs during the hospital stay, and 41 patients (13.6\%) experienced POI. HOSs were strongly associated with POI $(45.8 \%$ vs. $7.5 \%, \mathrm{P}<0.001)$. The all-cause readmission rate was $16.9 \%$, with $19(6.3 \%)$ and $20(6.6 \%)$ experiencing ileus and acute kidney injury, respectively. HOSs $(27.1 \%$ vs. $15.0 \%, \mathrm{P}=0.040)$ and POI $(34.1 \%$ vs. $14.2 \%, \mathrm{P}=0.002$ ) were associated with all-cause readmission, and $\mathrm{POI}$ was associated with readmission with ileus (17.1\% vs. $4.6 \%, \mathrm{P}=0.007)$. POI was an independent risk factor for all-cause readmission (adjusted odds ratio [OR], 2.640; 95\% confidence interval $[\mathrm{CI}], 1.162$ to $6.001 ; \mathrm{P}=0.020$ ) and readmission with ileus (adjusted $\mathrm{OR}=3.869 ; 95 \% \mathrm{CI} 1.387$ to 10.792; $\mathrm{P}=0.010)$.

Conclusion: POI was associated with readmission, particularly for subsequent ileus, in patients with diverting ileostomy. We should make efforts to reduce POI, such as strong control of HOSs, to prevent readmission.

Keywords: High-output stoma; Ileostomy; Postoperative ileus; Readmission; Rectal cancer

\section{INTRODUCTION}

Rectal cancer surgery has greatly advanced since the introduction

Received: Jun 1, 2020 • Revised: Jul 21, 2020 - Accepted: Aug 3, 2020 Correspondence to: Hyeong Rok Kim, M.D.

Department of Surgery, Chonnam National University Hwasun Hospital, Chonnam National University Medical School, 322 Seoyang-ro, Hwasuneup, Hwasun 58128, Korea

Tel: +82-61-379-7643, Fax: +82-61-379-7661

E-mail:drkhr@chonnam.ac.kr

ORCID: https://orcid.org/0000-0003-3747-1415

*Naa Lee and Soo Young Lee contributed equally as co-first authors. - The abstract of this article was presented at the Digestive Disease Week in San Diego, CA, USA on May 20, 2019.

(C) 2021 The Korean Society of Coloproctology

This is an open-access article distributed under the terms of the Creative Commons Attribution NonCommercial License (https://creativecommons.org/licenses/by-nc/4.0) which permits unrestricted noncommercial use, distribution, and reproduction in any medium, provided the original work is properly cited. of total mesorectal excision [1], resulting in improved oncologic outcomes and quality of life $[2,3]$. However, anastomotic leakage is still of great concern to many surgeons because of the increased probability of reoperation, morbidity, and mortality [4]. To protect the anastomosis, a diverting stoma is often constructed [5]. Although whether a diverting stoma reduces anastomotic leakage following rectal cancer surgery is still controversial, diverting stoma construction is thought to reduce the clinical severity of anastomotic leakage [6-8]. Because of their advantages during stoma reversal surgery, diverting ileostomies are usually recommended over colostomies [9].

However, a diverting ileostomy can result in substantial complications, such as bowel obstruction, parastomal hernia, prolapse, and dermatitis $[10,11]$. More importantly, significant morbidity, such as high-output stoma (HOS), dehydration, electrolyte imbalance, and acute kidney injury (AKI), may cause readmission after rectal cancer surgery with diverting ileostomy [12-19]. Readmission rates after ileostomy creation have been reported to range 
between $12.9 \%$ and $30 \%$ [12-18]. Although previous studies have reported the risk factors associated with readmission after ileostomy creation [12,15-18], there have been no solid conclusions.

Moreover, postoperative ileus (POI) is another common complication after rectal cancer surgery. POI results from an inflammatory response caused by bowel manipulation, which leads to prolonged inhibition of coordinated bowel activity [20]. Some previous studies reported that stoma formation may increase the risk of developing POI [20-22]. In addition, POI and early postoperative small bowel obstruction may lead to readmission from subsequent adhesive small bowel obstruction [20]. Considering that intermittent bowel obstruction may be one of the main causes of HOSs [23], we hypothesized that HOSs and POI may be closely related, which could result in readmission after diverting ileostomy construction.

Therefore, this study was designed to investigate the relationship between HOSs, POI, and readmission after rectal cancer surgery with diverting ileostomy.

\section{METHODS}

This study was approved by the Institutional Review Board of Chonnam National University Hwasun Hospital (CNUHH2019-036). We retrospectively reviewed a prospectively collected rectal cancer patient database and included 302 patients who underwent restorative resection with diverting ileostomy between January 2011 and December 2015. The informed consent of patients was waived because of the retrospective nature of the study. The included patients had tumors located 3 to $15 \mathrm{~cm}$ from the anal verge. Patients with distant metastasis or stage 4 or 5 chronic kidney disease were excluded.

Colonoscopy, abdominopelvic and chest computed tomography, pelvic magnetic resonance imaging, and serum carcinoembryonic antigen testing were conducted preoperatively. In all patients with locally advanced rectal cancer ( $\geq \mathrm{cT} 3$ or $\geq \mathrm{cN} 1$ ), neoadjuvant chemoradiotherapy (5,040 cGy in 28 fractions with 5-fluorouracil-based chemotherapy) was considered.

Following these procedures, radical surgery was performed according to general oncologic principles. When neoadjuvant chemoradiotherapy was administered, surgery was performed at 6 to 10 weeks after the completion of radiotherapy. A diverting ileostomy was created at the surgeon's discretion to protect the anastomosis. Before surgery, the diverting ileostomy site was marked by ostomy care nurses at $2 / 3$ of the distance between the right anterior superior iliac spine and umbilicus. A round incision was made in this area, the rectus muscles were widened, and the peritoneum was opened. Terminal ileum $20 \mathrm{~cm}$ away from the ileocecal valve was used for constructing ileostomy. In all patients, a support bridge was routinely placed under the diverting ileostomy. After abdominal wound closure, the ileostomy was matured with a transverse incision and the eversion technique.

For patients with pathologic stage II or higher tumors, adjuvant chemotherapy was considered. In cases of node-positive disease or circumferential resection margin involvement without neoadjuvant chemoradiotherapy, postoperative radiotherapy was also considered. All patients who underwent neoadjuvant chemoradiotherapy, regardless of the final pathologic stage, were recommended to receive adjuvant chemotherapy. Diverting ileostomy closure was considered at 2 months after initial surgery or, in cases treated with adjuvant chemotherapy, 1 to 2 months after completing the adjuvant treatment. Before diverting ileostomy closure, clinical anastomosis evaluation was performed through digital rectal examination or rigid proctoscopy.

HOSs were defined as stomas with $\geq 2,000 \mathrm{~mL} /$ day output [24]. POI was characterized by symptoms of nausea, vomiting, oral feeding intolerance, distended abdomen, or failure to pass flatus or bowel movements with clinical and radiologic findings within 7 days after surgery [25]. AKI was defined as serum creatinine levels of $\geq 1.4 \mathrm{mg} / \mathrm{dL}$ or an increase of serum creatinine levels of $\geq 0.3 \mathrm{mg} / \mathrm{dL}$ from the baseline value [26]. Postoperative complications were defined as complications occurred within 30 days after surgery. All unexpected readmissions during the period of maintaining ileostomy were included in the analysis.

Clinicopathologic data, such as patient sex, age, body mass index, American Society of Anesthesiologists physical status classification, hypertension, diabetes mellitus, smoking status, operative time and approach, tumor size, $\mathrm{T}$ and $\mathrm{N}$ stages, pre- and postoperative chemotherapy status, preoperative serum albumin level, and serum creatinine level on the day of discharge were collected. The included patients were divided into readmission and non-readmission groups. The readmission group was subdivided according to the reason for readmission (i.e., ileus or AKI). The predictive factors for readmission were analyzed.

Categorical variables were compared using the chi-square or Fisher exact test. We used multivariable logistic regression models to evaluate the predictive factors for readmission. The variables with $\mathrm{P}<0.1$ on univariable analysis were included in the multivariable logistic regression model. All results were considered clinically significant at $\mathrm{P}<0.05$. Statistical analyses were conducted using IBM SPSS Statistics vers. 22.0 (IBM Corp., Armonk, NY, USA).

\section{RESULTS}

Of the included patients, 104 (34.4\%) had postoperative complications after rectal cancer surgery with diverting ileostomy. Fortyone patients (13.6\%) had POI, and 14 (4.6\%) experienced anastomosis leakage (Table 1). Forty-eight patients (15.9\%) had HOSs during the hospital stay.

Table 2 shows the association between the clinicopathologic variables and POI. Men had a higher incidence of POI (17.2\% vs. $6.1 \%, \mathrm{P}=0.008$ ), whereas other clinicopathologic factors were not associated with POI. HOSs were highly associated with POI occurrence $(45.8 \%$ vs. $7.5 \%, \mathrm{P}<0.001)$. 


\section{Coloproctology Naa Lee, et al.}

Table 1. Postoperative complications

\begin{tabular}{lc}
\hline Complication & No. (\%) \\
\hline Postoperative ileus & $41(13.6)$ \\
Anastomotic leakage & $14(4.6)$ \\
Urinary retention & $11(3.6)$ \\
Urinary tract infection & $8(2.6)$ \\
Organ/space SSI (except leakage) & $7(2.3)$ \\
Wound infection & $7(2.3)$ \\
Pneumonia & $4(1.3)$ \\
Others & $21(7.0)$ \\
Total & $104(34.4)$ \\
\hline
\end{tabular}

SSI, surgical site infection.

Table 3. Cause of readmission

\begin{tabular}{lc}
\hline Cause & No. (\%) \\
\hline Acute kidney injury & $20(6.6)$ \\
lleus & $19(6.3)$ \\
Surgical site infection & $15(5.0)$ \\
Others & $5(1.7)$ \\
Total & $51(16.9)$ \\
\hline
\end{tabular}

Multiple complications in a patient were counted separately.

The all-cause readmission rate was $16.9 \%$. All readmissions occurred within 6 months after initial surgery. Of the readmitted patients, 20 (6.6\%) were diagnosed with AKI and 19 (6.3\%) experienced ileus (Table 3). Thirteen out of 48 patients (27.1\%) with HOSs were hospitalized again; 5 of whom were because of ileus, 5 were due to AKI, and the other 3 were due to surgical site infection. Table 4 shows the association between the clinicopathologic variables and readmission. $\mathrm{N}$ stage ( $24.5 \%$ vs. $12.8 \%, \mathrm{P}=0.009)$, HOSs $(27.1 \%$ vs. $15.0 \%, \mathrm{P}=0.040)$, and POI $(34.1 \%$ vs. $14.2 \%$, $\mathrm{P}=0.002$ ) were associated with all-cause readmission. Moreover, POI was associated with readmission due to ileus ( $17.1 \%$ vs. $4.6 \%$, $\mathrm{P}=0.007)$. Readmission due to AKI tended to be associated with diabetes mellitus ( $12.1 \%$ vs. $5.1 \%, \mathrm{P}=0.052)$, $\mathrm{T}$ stage $(9.2 \%$ vs. $3.1 \%, \mathrm{P}=0.034)$, and serum creatinine level at the time of discharge $(13.2 \%$ vs. $5.2 \%, \mathrm{P}=0.060)$.

Multivariable logistic regression analysis showed that advanced $\mathrm{N}$ stage (adjusted odds ratio [OR], 2.172; 95\% confidence interval [CI], 1.167 to 4.041; $\mathrm{P}=0.014$ ) and POI (adjusted OR, 2.640; 95\% CI, 1.162 to $6.001 ; \mathrm{P}=0.020$ ) were independent risk factors for allcause readmission (Table 5). The risk factors for readmission with ileus were open surgery (adjusted OR, 8.260; 95\% CI, 1.167 to 58.440; $\mathrm{P}=0.034$ ) and POI (adjusted OR, 3.869; 95\% CI, 1.387 to 10.792; $\mathrm{P}=0.010$ ). The risk factors for readmission due to AKI were diabetes mellitus (adjusted OR, 2.824; 95\% CI, 1.073 to 7.434; $\mathrm{P}=0.035$ ), advanced $\mathrm{T}$ stage (adjusted $\mathrm{OR}, 3.518 ; 95 \% \mathrm{CI}$, 1.120 to 11.054; $\mathrm{P}=0.031$ ), and serum creatinine levels of $>1.0$
Table 2. Association between clinicopathologic variables and postoperative ileus (POI)

\begin{tabular}{|c|c|c|c|}
\hline Variable & $\begin{array}{c}\text { POI (-) } \\
(\mathrm{n}=261)\end{array}$ & $\begin{array}{l}\text { POI (+) } \\
(n=41)\end{array}$ & P-value \\
\hline \multicolumn{4}{|l|}{ Sex } \\
\hline Male & $168(82.8)$ & $35(17.2)$ & 0.008 \\
\hline Female & $93(93.9)$ & $6(6.1)$ & \\
\hline \multicolumn{4}{|l|}{ Age (yr) } \\
\hline$<70$ & 168 (86.2) & 27 (13.8) & 0.853 \\
\hline$\geq 70$ & $93(86.9)$ & $14(13.1)$ & \\
\hline \multicolumn{4}{|c|}{ Body mass index $\left(\mathrm{kg} / \mathrm{m}^{2}\right)$} \\
\hline$<25$ & $196(86.0)$ & $32(14.0)$ & 0.683 \\
\hline$\geq 25$ & $65(87.8)$ & 9 (12.2) & \\
\hline \multicolumn{4}{|c|}{ ASA PS classification } \\
\hline |, || & $245(86.0)$ & $40(14.0)$ & 0.701 \\
\hline III, IV & $13(92.9)$ & $1(7.1)$ & \\
\hline \multicolumn{4}{|l|}{ Hypertension } \\
\hline No & $160(88.4)$ & $21(11.6)$ & 0.221 \\
\hline Yes & $101(83.5)$ & $20(16.5)$ & \\
\hline \multicolumn{4}{|l|}{ Diabetes mellitus } \\
\hline No & $206(87.3)$ & $30(12.7)$ & 0.407 \\
\hline Yes & 55 (83.3) & $11(16.7)$ & \\
\hline \multicolumn{4}{|l|}{ Smoking status } \\
\hline Smoker & $237(87.1)$ & $35(12.9)$ & 0.267 \\
\hline Nonsmoker & $24(80.0)$ & $6(20.0)$ & \\
\hline \multicolumn{4}{|c|}{ Previous abdominal surgery } \\
\hline No & $217(85.8)$ & $36(14.2)$ & 0.452 \\
\hline Yes & 44 (89.8) & 5 (10.2) & \\
\hline \multicolumn{4}{|c|}{ Operative time (min) } \\
\hline$<180$ & $167(87.4)$ & $24(12.6)$ & 0.501 \\
\hline$\geq 180$ & $94(84.7)$ & $17(15.3)$ & \\
\hline \multicolumn{4}{|c|}{ Operative approach } \\
\hline Open & $3(60.0)$ & $2(40.0)$ & 0.138 \\
\hline Laparoscopy & 258 (86.9) & $39(13.1)$ & \\
\hline \multicolumn{4}{|l|}{ Tumor size (cm) } \\
\hline$<5$ & $214(86.3)$ & $34(13.7)$ & 0.957 \\
\hline$\geq 5$ & $43(86.0)$ & $7(14.0)$ & \\
\hline \multicolumn{4}{|c|}{ Preoperative chemoradiotherapy } \\
\hline Not performed & $69(87.3)$ & $10(12.7)$ & 0.782 \\
\hline Performed & $192(86.1)$ & $31(13.9)$ & \\
\hline \multicolumn{4}{|c|}{ High-output stoma } \\
\hline No & $235(92.5)$ & $19(7.5)$ & $<0.001$ \\
\hline Yes & $26(54.2)$ & $22(45.8)$ & \\
\hline \multicolumn{4}{|c|}{ Albumin (g/dL), preoperative } \\
\hline$\leq 3.5$ & $13(86.7)$ & $2(13.3)$ & 0.999 \\
\hline$>3.5$ & $248(86.4)$ & 39 (13.6) & \\
\hline
\end{tabular}

Values are presented as number (\%).

ASA, American Society of Anesthesiologists; PS, physical status. 
Table 4. Association between clinicopathologic variables and readmission

\begin{tabular}{|c|c|c|c|c|c|c|c|}
\hline \multirow{2}{*}{ Variable } & \multirow{2}{*}{$\begin{array}{l}\text { No readmission } \\
\quad(n=251)\end{array}$} & \multicolumn{6}{|c|}{ Readmission } \\
\hline & & All $(n=51)$ & P-value & Ileus $(n=19)$ & P-value & AKI $(n=20)$ & P-value \\
\hline \multicolumn{8}{|l|}{ Sex } \\
\hline Male & $164(80.8)$ & 39 (19.2) & 0.123 & $16(7.9)$ & 0.103 & $16(7.9)$ & 0.208 \\
\hline Female & $87(87.9)$ & $12(12.1)$ & & $3(3.0)$ & & $4(4.0)$ & \\
\hline \multicolumn{8}{|l|}{ Age (yr) } \\
\hline$<70$ & $164(84.1)$ & $31(15.9)$ & 0.535 & $12(6.2)$ & 0.894 & $12(6.2)$ & 0.658 \\
\hline$\geq 70$ & 87 (81.3) & 20 (18.7) & & $7(6.5)$ & & $8(7.5)$ & \\
\hline \multicolumn{8}{|c|}{ Body mass index $\left(\mathrm{kg} / \mathrm{m}^{2}\right)$} \\
\hline$<25$ & $186(81.6)$ & $42(18.4)$ & 0.212 & $15(6.6)$ & 0.999 & $16(7.0)$ & 0.628 \\
\hline$\geq 25$ & 65 (87.8) & 9 (12.2) & & $4(5.4)$ & & $4(5.4)$ & \\
\hline \multicolumn{8}{|c|}{ ASA PS classification } \\
\hline I, || & 238 (83.5) & 47 (16.5) & 0.711 & $19(6.7)$ & 0.999 & $18(6.3)$ & 0.239 \\
\hline III, IV & $11(78.6)$ & 3 (21.4) & & $0(0.0)$ & & $2(14.3)$ & \\
\hline \multicolumn{8}{|l|}{ Hypertension } \\
\hline No & $150(82.9)$ & $31(17.1)$ & 0.892 & $11(6.1)$ & 0.851 & $12(6.6)$ & 0.995 \\
\hline Yes & $101(83.5)$ & $20(16.5)$ & & $8(6.6)$ & & $8(6.6)$ & \\
\hline \multicolumn{8}{|l|}{ Diabetes mellitus } \\
\hline No & $197(83.5)$ & 39 (16.5) & 0.751 & $14(5.9)$ & 0.576 & $12(5.1)$ & 0.052 \\
\hline Yes & $54(81.8)$ & $12(18.2)$ & & $5(7.6)$ & & $8(12.1)$ & \\
\hline \multicolumn{8}{|l|}{ Smoking status } \\
\hline Smoker & $226(83.1)$ & $46(16.9)$ & 0.973 & $16(5.9)$ & 0.417 & $19(7.0)$ & 0.705 \\
\hline Nonsmoker & $25(83.3)$ & $5(16.7)$ & & $3(10.0)$ & & $1(3.3)$ & \\
\hline \multicolumn{8}{|c|}{ Previous abdominal surgery } \\
\hline No & $210(83.0)$ & $43(17.0)$ & 0.909 & $16(6.3)$ & 0.999 & $15(5.9)$ & 0.341 \\
\hline Yes & 41 (83.7) & $8(16.3)$ & & $3(6.1)$ & & $5(10.2)$ & \\
\hline \multicolumn{8}{|c|}{ Operative time (min) } \\
\hline$<180$ & $162(84.8)$ & $29(15.2)$ & 0.300 & $9(4.7)$ & 0.138 & $12(6.3)$ & 0.755 \\
\hline$\geq 180$ & 89 (80.2) & $22(19.8)$ & & $10(9.0)$ & & $8(7.2)$ & \\
\hline \multicolumn{8}{|c|}{ Operative approach } \\
\hline Open & $3(60.0)$ & $2(40.0)$ & 0.199 & $2(40.0)$ & 0.034 & $1(20.0)$ & 0.292 \\
\hline Laparoscopy & $248(83.5)$ & $49(16.5)$ & & $17(5.7)$ & & $19(6.4)$ & \\
\hline \multicolumn{8}{|l|}{ Tumor size $(\mathrm{cm})$} \\
\hline$<5$ & 207 (83.5) & $41(16.5)$ & 0.553 & $16(6.5)$ & 0.999 & $16(6.5)$ & 0.756 \\
\hline$\geq 5$ & $40(80.0)$ & $10(20.0)$ & & $3(6.0)$ & & $4(8.0)$ & \\
\hline \multicolumn{8}{|l|}{ T stage } \\
\hline $0,1,2$ & $112(86.8)$ & $17(13.2)$ & 0.137 & $6(4.7)$ & 0.311 & $4(3.1)$ & 0.034 \\
\hline 3,4 & $139(80.3)$ & $34(19.7)$ & & $13(7.5)$ & & $16(9.2)$ & \\
\hline \multicolumn{8}{|l|}{ N stage } \\
\hline 0 & $171(87.2)$ & $25(12.8)$ & 0.009 & $10(5.1)$ & 0.247 & $10(5.1)$ & 0.148 \\
\hline 1,2 & 80 (75.5) & $26(24.5)$ & & $9(8.5)$ & & $10(9.4)$ & \\
\hline \multicolumn{8}{|c|}{ Preoperative chemoradiotherapy } \\
\hline Not performed & 62 (78.5) & 17 (21.5) & 0.201 & $4(5.1)$ & 0.789 & $5(6.3)$ & 0.903 \\
\hline Performed & 189 (84.8) & $34(15.2)$ & & $15(6.7)$ & & $15(6.7)$ & \\
\hline
\end{tabular}


Table 4. Continued

\begin{tabular}{|c|c|c|c|c|c|c|c|}
\hline \multirow{2}{*}{ Variable } & \multirow{2}{*}{$\begin{array}{l}\text { No readmission } \\
\quad(n=251)\end{array}$} & \multicolumn{6}{|c|}{ Readmission } \\
\hline & & All $(n=51)$ & P-value & Ileus $(n=19)$ & P-value & AKI $(n=20)$ & P-value \\
\hline \multicolumn{8}{|c|}{ Postoperative chemo(radio)therapy } \\
\hline Not performed & 45 (83.3) & $9(16.7)$ & 0.962 & $1(1.9)$ & 0.215 & $4(7.4)$ & 0.765 \\
\hline Performed & $206(83.1)$ & $42(16.9)$ & & $18(7.3)$ & & $16(6.5)$ & \\
\hline \multicolumn{8}{|c|}{ High-output stoma } \\
\hline No & $216(85.0)$ & $38(15.0)$ & 0.040 & $14(5.5)$ & 0.200 & $15(5.9)$ & 0.336 \\
\hline Yes & 35 (72.9) & $13(27.1)$ & & $5(10.4)$ & & $5(10.4)$ & \\
\hline \multicolumn{8}{|c|}{ Postoperative ileus } \\
\hline No & $224(85.8)$ & 37 (14.2) & 0.002 & $12(4.6)$ & 0.007 & $15(5.7)$ & 0.166 \\
\hline Yes & $27(65.9)$ & $14(34.1)$ & & $7(17.1)$ & & $5(12.2)$ & \\
\hline \multicolumn{8}{|c|}{ Albumin (g/dL), preoperative } \\
\hline$\leq 3.5$ & $13(86.7)$ & $2(13.3)$ & 0.999 & $1(6.7)$ & 0.999 & $2(13.3)$ & 0.261 \\
\hline$>3.5$ & $238(82.9)$ & 49 (17.1) & & $18(6.3)$ & & $18(6.3)$ & \\
\hline \multicolumn{8}{|c|}{ Creatinine $(\mathrm{mg} / \mathrm{dL})$, day of discharge } \\
\hline$\leq 1.0$ & $209(83.9)$ & $40(16.1)$ & 0.408 & $16(6.4)$ & 0.999 & $13(5.2)$ & 0.060 \\
\hline$>1.0$ & $42(79.2)$ & $11(20.8)$ & & $3(5.7)$ & & $7(13.2)$ & \\
\hline
\end{tabular}

Values are presented as number (\%).

AKI, acute kidney injury; ASA, American Society of Anesthesiologists; PS, physical status.

$\mathrm{mg} / \mathrm{dL}$ on the day of discharge (adjusted OR, 2.881; 95\% CI, 1.063 to $7.809 ; \mathrm{P}=0.038$ ) (Table 5).

\section{DISCUSSION}

In the present study, we investigated the association between POI, HOSs, and readmission after rectal cancer surgery with diverting ileostomy. We found that POI following diverting ileostomy construction was associated with HOSs, which resulted in subsequent ileus and readmission.

HOSs have been reported to occur in $16 \%$ of patients with stomas $[23,27]$. However, there is no agreed definition for HOSs, which have been defined as having outputs of $\geq 1,000$ to 2,000 $\mathrm{mL} /$ day [23]. In this study, we used $2,000 \mathrm{~mL} /$ day as a cut-off value similar to previous studies [23, 24]. An HOS can cause fluid depletion, electrolyte imbalance, and micronutrient disturbance, resulting in delayed recovery and increased hospital costs. Therefore, it is very important to properly manage HOSs [23]. Some authors have reported that HOS presence was an independent risk factor for readmission after stoma creation [18], whereas other authors have not found a significant association between HOSs and readmission $[13,14]$. In the present study, HOSs were associated with all-cause readmission in the univariable, but not multivariable, analysis. This result was observed because HOSs and POI were highly correlated, and POI was a more significant predictive factor for all-cause readmission (Table 5). Although HOS was not identified as an independent predictive factor of readmission in multivariable analysis, when we performed a multi-
Table 5. Multivariable analysis of the predictive factors for readmission

\begin{tabular}{llc}
\hline Factor & Adjusted OR (95\% Cl) & P-value \\
\hline All-cause readmission & & \\
N1 or N2 stage & $2.172(1.167-4.041)$ & 0.014 \\
High-output stoma & $1.407(0.619-3.199)$ & 0.415 \\
Postoperative ileus & $2.640(1.162-6.001)$ & 0.020 \\
Readmission with ileus & & \\
Open surgery & $8.260(1.167-58.440)$ & 0.034 \\
Postoperative ileus & $3.869(1.387-10.792)$ & 0.010 \\
Readmission with AKI & & \\
Diabetes mellitus & $2.824(1.073-7.434)$ & 0.035 \\
T3 or T4 stage & $3.518(1.120-11.054)$ & 0.031 \\
Creatinine $>1.0$ mg/dL, day of discharge & $2.881(1.063-7.809)$ & 0.038 \\
\hline
\end{tabular}

$\mathrm{OR}$, odds ratio; $\mathrm{Cl}$, confidence interval; $\mathrm{AKI}$, acute kidney injury.

variable analysis after excluding POI, HOS was proved to be an independent predictive factor for readmission (OR, 2.058; 95\% CI, 1.011 to 4.290; $\mathrm{P}=0.048$ ) (data not shown). This might be derived from some correlations between HOS and POI in clinical practice, although multicollinearity was not observed between HOS and POI (variance inflation factor, 2.201). Considering our relatively small number of patients with HOSs and POI, this should be analyzed in more detail with a larger number of patients.

Gastrointestinal inflammation caused by bowel manipulation 
combined with other factors, such as fluid overloading and opioid use, is the key mechanism underlying POI development [20, 28]. Several risk factors have been suggested for POI development, such as total colectomy, comorbidities, urgent operation, and long operative time [20,21]. Moreover, some studies have reported that stoma formation was an independent risk factor for POI after colorectal surgery $[21,22]$. One possible explanation for the association between stoma formation and POI is that extensive ileocolic manipulation for ileostomy construction may cause more tissue inflammation and, thus, lead to POI development. Another possible mechanism of POI development is partial obstruction caused by the rotation and kinking associated with ileostomy formation [20]. Such partial obstruction can also trigger HOSs, which may be a plausible explanation for the high correlation between POI and HOSs observed in the present study.

Previous studies have reported that dehydration is the main cause of readmission after ileostomy creation, and several risk factors for readmission, such as comorbidity, loop stoma, longer hospital stay, the use of diuretics, antidiarrheals, and the laparoscopic approach, preoperative chemoradiotherapy, older age, and HOSs, have been suggested [12-18]. In this study, we found that POI was an independent risk factor for all-cause readmission and readmission related to ileus (Table 5). This observation is consistent with that of a previous study in which early postoperative small bowel obstruction was found to be an independent risk factor for subsequent adhesive ileus in patients undergoing colectomy [20]. As previously stated, HOSs and POI were highly correlated with small bowel partial obstruction, which could cause subsequent readmission, particularly due to ileus.

In the present study, the risk factors for readmission with AKI were diabetes mellitus, advanced $\mathrm{T}$ stage, and serum creatinine levels of $>1.0 \mathrm{mg} / \mathrm{dL}$ on the day of discharge. Considering that patients with ileostomy are at high risk of dehydration [12-18], AKI in patients with diverting ileostomy may be triggered if basal renal function is poor, such as in patients with diabetes mellitus or high serum creatinine levels after ileostomy formation. Therefore, in such patients, HOSs should be managed more carefully to prevent AKI.

Appropriate HOS management is essential in patients with diverting stomas. Primarily, patients with HOSs should restrict hypotonic or hypertonic fluids to $<1,000 \mathrm{~mL} /$ day [23]. If patients with HOSs feel thirsty, then they should be provided with oral glucose-saline solutions [23]. In addition, careful nutritional status assessment and nutrient deficiency replacement should be performed, and antisecretory or antidiarrheal medication and psychological support are also important [23, 29]. Using this systematic and multidisciplinary approach, the risk of dehydration and readmission can be decreased in patients with HOSs.

The present study has several limitations. First, because this study was retrospective and limited to a single center, it is difficult to assess the generalizability of our findings concerning readmission risk factors. Second, the multivariable logistic regression models were somewhat heavy because of the relatively small number of included patients. Third, the number of patients with HOSs was relatively small $(n=48)$ to prove the relationship between HOS and readmission due to ileus or AKI. Further studies with large number of patients are needed. Nevertheless, our study suggests a possible relationship between HOS formation and POI development, which could result in the readmission of patients with diverting ileostomy.

In conclusion, HOSs were associated with POI, resulting in readmission, particularly for subsequent ileus, in patients with diverting ileostomy. Efforts should be made to reduce POI to prevent readmission. Patients with HOSs should be managed more carefully to limit the incidence of POI.

\section{CONFLICT OF INTEREST}

No potential conflict of interest relevant to this article was reported.

\section{REFERENCES}

1. Heald RJ, Husband EM, Ryall RD. The mesorectum in rectal cancer surgery: the clue to pelvic recurrence? Br J Surg 1982;69:613-6.

2. Kim NK, Kim YW, Min BS, Lee KY, Sohn SK, Cho CH. Operative safety and oncologic outcomes of anal sphincter-preserving surgery with mesorectal excision for rectal cancer: 931 consecutive patients treated at a single institution. Ann Surg Oncol 2009;16: 900-9.

3. Shirouzu K, Ogata Y, Araki Y. Oncologic and functional results of total mesorectal excision and autonomic nerve-preserving operation for advanced lower rectal cancer. Dis Colon Rectum 2004;47: $1442-7$.

4. McDermott FD, Heeney A, Kelly ME, Steele RJ, Carlson GL, Winter DC. Systematic review of preoperative, intraoperative and postoperative risk factors for colorectal anastomotic leaks. $\mathrm{Br} J$ Surg 2015;102:462-79.

5. Hanna MH, Vinci A, Pigazzi A. Diverting ileostomy in colorectal surgery: when is it necessary? Langenbecks Arch Surg 2015;400: 145-52.

6. Marusch F, Koch A, Schmidt U, Geibetaler S, Dralle H, Saeger $\mathrm{HD}$, et al. Value of a protective stoma in low anterior resections for rectal cancer. Dis Colon Rectum 2002;45:1164-71.

7. Sciuto A, Merola G, de Palma GD, Sodo M, Pirozzi F, Bracale UM, et al. Predictive factors for anastomotic leakage after laparoscopic colorectal surgery. World J Gastroenterol 2018;24:2247-60.

8. Pisarska M, Gajewska N, Małczak P, Wysocki M, Witowski J, Torbicz G, et al. Defunctioning ileostomy reduces leakage rate in rectal cancer surgery: systematic review and meta-analysis. Oncotarget 2018;9:20816-25.

9. Li L, Lau KS, Ramanathan V, Orcutt ST, Sansgiry S, Albo D, et al. Ileostomy creation in colorectal cancer surgery: risk of acute kidney injury and chronic kidney disease. J Surg Res 2017;210:204- 
12.

10. Phatak UR, Kao LS, You YN, Rodriguez-Bigas MA, Skibber JM, Feig BW, et al. Impact of ileostomy-related complications on the multidisciplinary treatment of rectal cancer. Ann Surg Oncol 2014; 21:507-12.

11. Caricato M, Ausania F, Ripetti V, Bartolozzi F, Campoli G, Coppola R. Retrospective analysis of long-term defunctioning stoma complications after colorectal surgery. Colorectal Dis 2007;9:55961.

12. Messaris E, Sehgal R, Deiling S, Koltun WA, Stewart D, McKenna $\mathrm{K}$, et al. Dehydration is the most common indication for readmission after diverting ileostomy creation. Dis Colon Rectum 2012;55: 175-80.

13. Hayden DM, Pinzon MC, Francescatti AB, Edquist SC, Malczewski MR, Jolley JM, et al. Hospital readmission for fluid and electrolyte abnormalities following ileostomy construction: preventable or unpredictable? J Gastrointest Surg 2013;17:298-303.

14. Paquette IM, Solan P, Rafferty JF, Ferguson MA, Davis BR. Readmission for dehydration or renal failure after ileostomy creation. Dis Colon Rectum 2013;56:974-9.

15. Fish DR, Mancuso CA, Garcia-Aguilar JE, Lee SW, Nash GM, Sonoda $\mathrm{T}$, et al. Readmission after ileostomy creation: retrospective review of a common and significant event. Ann Surg 2017;265: 379-87.

16. Li W, Stocchi L, Cherla D, Liu G, Agostinelli A, Delaney CP, et al. Factors associated with hospital readmission following diverting ileostomy creation. Tech Coloproctol 2017;21:641-8.

17. Chen SY, Stem M, Cerullo M, Canner JK, Gearhart SL, Safar B, et al. Predicting the risk of readmission from dehydration after ileostomy formation: the dehydration readmission after ileostomy prediction score. Dis Colon Rectum 2018;61:1410-7.

18. Justiniano CF, Temple LK, Swanger AA, Xu Z, Speranza JR, Cellini C, et al. Readmissions with dehydration after ileostomy creation: rethinking risk factors. Dis Colon Rectum 2018;61:1297305.

19. Gessler B, Haglind E, Angenete E. A temporary loop ileostomy affects renal function. Int J Colorectal Dis 2014;29:1131-5.

20. Lee SY, Park KJ, Ryoo SB, Oh HK, Choe EK, Heo SC. Early postoperative small bowel obstruction is an independent risk factor for subsequent adhesive small bowel obstruction in patients undergoing open colectomy. World J Surg 2014;38:3007-14.

21. Chapuis PH, Bokey L, Keshava A, Rickard MJ, Stewart P, Young CJ, et al. Risk factors for prolonged ileus after resection of colorectal cancer: an observational study of 2400 consecutive patients. Ann Surg 2013;257:909-15.

22. Reichert M, Weber C, Pons-Kühnemann J, Hecker M, Padberg W, Hecker A. Protective loop ileostomy increases the risk for prolonged postoperative paralytic ileus after open oncologic rectal resection. Int J Colorectal Dis 2018;33:1551-7.

23. Mountford CG, Manas DM, Thompson NP. A practical approach to the management of high-output stoma. Frontline Gastroenterol 2014;5:203-7.

24. Baker ML, Williams RN, Nightingale JM. Causes and management of a high-output stoma. Colorectal Dis 2011;13:191-7.

25. Gero D, Gié O, Hübner M, Demartines N, Hahnloser D. Postoperative ileus: in search of an international consensus on definition, diagnosis, and treatment. Langenbecks Arch Surg 2017;402: 149-58.

26. Van Manen L, Handgraaf HJ, Diana M, Dijkstra J, Ishizawa T, Vahrmeijer AL, et al. A practical guide for the use of indocyanine green and methylene blue in fluorescence-guided abdominal surgery. J Surg Oncol 2018;118:283-300.

27. Kristensen K, Qvist N. The acute effect of loperamide on ileostomy output: a randomized, double-blinded, placebo-controlled, crossover study. Basic Clin Pharmacol Toxicol 2017;121:493-8.

28. Bragg D, El-Sharkawy AM, Psaltis E, Maxwell-Armstrong CA, Lobo DN. Postoperative ileus: recent developments in pathophysiology and management. Clin Nutr 2015;34:367-76.

29. Arenas Villafranca JJ, López-Rodríguez C, Abilés J, Rivera R, Gándara Adán N, Utrilla Navarro P. Protocol for the detection and nutritional management of high-output stomas. Nutr J 2015;14:45. 